Um diese Fragen zu beantworten, hat Flaig nicht nur das historische Material bemüht, sondern auch Debatten aus der modernen ökonomischen Entscheidungstheorie, Theorien der deliberativen Demokratie und Thesen von John Rawls in seine Überlegungen mit eingeflochten. Als Stärke des Buches ist anzusehen, dass Flaig auf die aufgelisteten Fragen keine einheitlichen Antworten geben kann und will; vielmehr belässt er es bei dem historisch informierten Facettenreichtum der Voraussetzungen, Funktionsweisen und Dynamiken der Mehrheitsregel. Sein Buch lädt den Leser dazu ein, zu eigenen Antworten auf die aufgeworfenen Fragen zu gelangen.

Je mehr man sich in den Band vertieft, desto stärker gewinnt man im Übrigen den Eindruck, dass sich die Anhängerschaft des Verfassers für die Mehrheitsregel möglicherweise nicht allein dem wortreich zur Schau gestellten politischen Egalitarismus der Athener (bei einer zuweilen befremdlich anmutenden souveränen Ignoranz gegenüber dem radikalen Anti-Egalitarismus in der sozialen Sphäre) verdankt. Denn zum einen gehört nach Meinung von Flaig nichts Geringeres als die Entstehung der Wissenschaft zu einer der notwendigen Folgen der Mehrheitsregel in Griechenland. Und zum anderen hebt er mehrfach positiv hervor, wie die mit der Mehrheitsregel verbundene Senkung der politischen Entscheidungskosten Athen an Effizienz und politischer Schlag- und Expansionskraft gewinnen ließ.

Wie dem auch sei - der Autor hat in der Einleitung nicht zu viel versprochen: Das Buch bietet viel bislang nicht zu Lesendes und Anregendes zum Thema Mehrheitsregel und sei deshalb zur Lektüre unbedingt empfohlen.

Hubertus Buchstein

\title{
Regieren in Theorie, Praxis und Vergleich - gelungene Festschrift
}

Egner, Björn, Michael Haus und Georgios Terizakis (Hrsg.): Regieren. Festschrift für Hubert Heinelt, VS Verlag für Sozialwissenschaften, Wiesbaden 2012, 563 Seiten, € 69,95.

Regieren ist ein komplexer, interessengeleiteter, langsamer und enttäuschungsanfälliger Prozess. „Ungenügender Erfolg“, so Friedbert W. Rüb, ist eine unvermeidbare Begleiterscheinung moderner Staatstätigkeit (S. 131). Werner Jann hat es Mitte der 1990er Jahre konzise formuliert: „Regierungen steuern nicht, sie intervenieren.“

Fünf Dimensionen legen sich wie Jahresringe um den Begriff des Regierens: In klassischer Perspektive geht Regieren von der Steuerungsfähigkeit des politisch-administrativen Systems aus. Angesichts steigender gesellschaftlicher Komplexität stehen zweitens verhandlungsdemokratische Arrangements, die auch von Regierungen unterstützt werden. Drittens - und eigentlich an zentraler Stelle - geht es beim Regieren um „policy making“, mithin um die zyklische Abfolge einzelner Schritte im Politikprozess. Heute werden viertens westliche Regierungssysteme vielfach mit dem Etikett der Postdemokratie versehen, das eine ganze Bandbreite an Defiziten auf den Begriff zu bringen versucht. Und fünftens firmiert modernes Regieren unter Governance und soll dabei komplexe Mischformen aus staatlicher Regulierung und gesellschaftlicher Selbststeuerung bedeuten.

Alle diese genannten Aspekte werden im vorliegenden Sammelband aus unterschiedlichen Perspektiven angesprochen. Konzipiert ist das Buch als Festschrift für Hubert Heinelt, der 
im August 2012 seinen 60. Geburtstag begangen hat. In durchaus „provokativer“ Absicht (S. 10), so die Herausgeber im Vorwort, trägt der Band diesen äußerst knappen Titel. „Regieren“ ist ein „wichtiger Bezugspunkt“ und zugleich „die Klammer“ (S. 10) für all jene Themen, die Heinelt in seinem wissenschaftlichen Schaffen erforscht hat. Drei große Abschnitte gliedern den Band: Nach einführender Diskussion von Theorien, Konzepten und Ideen werden einzelne Politikfelder beleuchtet; ein dritter Teil widmet sich der Regierungstätigkeit in vergleichender Perspektive.

Regieren als genuiner Erklärungsgegenstand formuliert im Subtext die Frage nach der Problemlösungsfähigkeit des politisch-administrativen Systems. Zwar fallen die jeweiligen institutionalisierten Arrangements des Regierens einschließlich ihrer normativen Konzeptionen recht unterschiedlich aus (wie zum Beispiel hierarchische Steuerung, Netzwerke, advocacy coalitions etc.), doch bleibt am Ende immer - in den Worten von Thomas R. Dyedie Frage „what governments do, why they do it and what difference it makes“.

So fragt Friedbert W. Rüb zunächst, was Regierungen eigentlich machen, wenn sie regieren. Anhand der principal-agent-Theorie zeigt Rüb, dass Regierungen vielfach damit beschäftigt sind, Delegationsprozesse zu organisieren, aber diese zugleich auch zu überwachen. Um Kontrollverlusten (so genannte agency loss oder agency capture) entgegenzuwirken, liegen in Gestalt von Koalitionsverträgen oder des Koalitionsausschusses adäquate Instrumente vor. Doch die „Verexekutivierung des Regierens“ (S. 130), mithin eine starke Zentralregierung, erschwerte effektive Kontrolle.

Dass Regierungstätigkeit stets auch normativ-konzeptionell eingebettet ist, zeigt Michael Haus in seiner Gegenüberstellung des Postdemokratie- und Governance-Diskurses. Folgt man den Adepten postdemokratischer Zustände, dann wandern demokratische Entscheidungen aus staatlichen Institutionen aus und werden zunehmend von abgeschlossenen Elitenkartellen getroffen. Dieser postdemokratische Zweig der aktuellen Debatte ist von „Melancholie, Verweigerung und Protest“ (S. 136) grundiert. Governance-Konzepte hingegen spiegeln ein „posthierarchisches Verständnis des Regierens“ und gleichzeitig die „Verflüssigung und Zerfaserung der Grenzen zwischen Staat, Markt und Zivilgesellschaft“ (S. 138). Heute wird Postdemokratie „als treffliche Beschreibung der gegenwärtigen politischen Grundstimmung in Anspruch genommen“ (S. 141). Abschließend bezeichnet Michael Haus jedoch die Annahme, dass sich die „wahren“ demokratischen Zustände immer mehr auflösen, als „nicht überzeugend“ (S. 151).

In den traurigen Abgesang, der seine Energie aus postdemokratischen Verfallsbildern bezieht, stimmt Heidrun Abromeit ein. Ihren Ansichten muss man nicht folgen. Sie stellt die „Privatisierung der Politik“ (S. 106) fest, zu deren Verschleierung der Governance-Begriff verwendet werde. Damit wird Zynismus zum dominanten Prinzip des Regierens, und wo politische Steuerung durch kooperative Handlungsformen vollzogen wird, macht sich in Wahrheit Steuerungsversagen breit. Auch um den modernen Politiker-Typus sei es nicht gut bestellt: „Er will nur noch gewinnen, nicht gestalten; er will die Vorteile der Macht genießen, ohne entscheiden zu müssen“ (S. 105).

Aus realistisch-negativer Sicht plädiert Detlef Sack dafür, Regieren und Ironie im Zusammenhang zu denken. Bestenfalls führe Regieren zu gesellschaftlichen Problemlösungen, im schlechten Fall scheitert Politik jedoch. Aus dieser „immanenten Enttäuschungsoffenheit“ plädiert Sack für eine Haltung des Bürgers, die er „als Fähigkeit zur Ironie und als Ambiguitätstoleranz" (S. 161) beschreibt. Doch ist es nicht nur die oftmals begrenzte Reichweite von Politik, die Enttäuschungen produziert; der Bürger selbst formuliert hohe Beteiligungs- 
ansprüche und tritt damit als zivilgesellschaftlicher Mitregent auf. Damit liegen die Hürden des Scheiterns auch bei ihm selbst.

Eine Reihe der vorliegenden Kapitel rekurriert auf die unterschiedlichen Variationen von Governance. Es ist dieser bisweilen schillernde Begriff, der die vielfachen Metamorphosen politischer Steuerung widerspiegelt. Politische Steuerung vollzieht sich „prozedural“ (S. 247); Regieren lässt sich mithin auch als Steuerung durch Verfahren auffassen. In den Worten Dietrich Fürsts handelt es sich um „intrinsische Steuerung“ (S. 372), bei der Verhandlungen und Kooperationsbeziehungen zwischen Politik und Adressaten an Bedeutung gewinnen.

Die insgesamt 31 Beiträge fügen sich zu einem gelungenen Sammelband zusammen. Entstanden ist ein dicht konzipiertes Werk, dem eine in dreifacher Hinsicht breite Leserschaft zu wünschen ist: Zu empfehlen ist es all jenen, die etwas über modernes Regieren erfahren möchten genauso wie jenen, die neugierig sind auf das weite Themenfeld Hubert Heinelts. Schließlich eignet sich die Festschrift auch als anspruchsvolle Basislektüre für politikwissenschaftliche Hauptseminare.

Helge F. Jani

\section{Regieren in Multiperspektivität - schnelle Einblicke, neue Entwicklungen}

Korte, Karl-Rudolf und Timo Grunden (Hrsg.): Handbuch Regierungsforschung, VS Verlag für Sozialwissenschaften, Wiesbaden 2013, 434 Seiten, € 34,99.

Eine ganze Reihe von Handbüchern erschließen mittlerweile die Sozial- und Politikwissenschaften. Der hier anzuzeigende Sammelband zum Begriff des Regierens setzt sich mit 434 Seiten bereits optisch von anderen Titeln ab: Das Handbuch Parteienforschung oder das Handbuch Wahlforschung präsentieren ihr Feld in fast doppeltem Umfang. ${ }^{1}$ Was kann das Handbuch Regierungsforschung vor diesem Hintergrund leisten?

Die Herausgeber von der Universität Duisburg-Essen nähern sich dem „unbestimmten“ Begriff des Regierens (S. 11) in seiner Vielschichtigkeit. Sie möchten die Vielfalt des Gegenstandsbereichs systematisch darstellen und damit sowohl traditionelle Aspekte als auch jüngere Forschungsansätze integrieren (S. 15). Die Governance-Perspektive des Regierens ist dabei nur ein Teil des Forschungsfeldes, was eine Abgrenzung zum Handbuch Governance schafft. ${ }^{2}$ Korte und Grunden nehmen in der Systematisierung der Beiträge drei Schwerpunktsetzungen vor. Die Grundlage bildet - erstens - zumeist der klassische Blick auf die zentralen Institutionen des politischen Entscheidungsprozesses im Zusammenspiel von Parlament und Regierung. Die „politische Führung“ (S. 16) in Form einer demokratischen Regierung bildet letztlich den „inneren Kern“ (S. 16) des Handbuches, um den sich alle Beiträge bewegen (zum Beispiel RolfG. Heinze zu Handlungsspielräumen oder Karl-Rudolf Korte zum

1 Vgl. Oskar Niedermayer (Hrsg.), Handbuch Parteienforschung, Wiesbaden 2013; Jürgen Falter I Harald Schoen (Hrsg.), Handbuch Wahlforschung, Wiesbaden 2005.

2 Vgl. Arthur Benz / Susanne Lütz / Uwe Schimank / Georg Simonis (Hrsg.), Handbuch Governance, Wiesbaden 2007. 